\title{
Looking for life on Mars with the Rosalind Franklin rover: the PanCam instrument
}

A. Coates, M. Carter, C. Leff, A. Griffiths, N. Schmitz, et al.

A. J. Coates, M. Carter, C. E. Leff, A. D. Griffiths, N. Schmitz, R. Jaumann, J.L. Josset, G. Paar, M. Gunn, B. Whiteside, T. Hunt, B. Yu, T. Theodorou, "Looking for life on Mars with the Rosalind Franklin rover: the PanCam instrument," Proc. SPIE 11576, Hyperspectral Imaging and Applications, 1157602 (4 October 2020); doi: 10.1117/12.2584841

SPIE. Event: SPIE Photonex and Vacuum Expo, 2020, Online Only 


\title{
Looking for life on Mars withethepesasalind Franklin rover: the PanCam instrument
}

\author{
A.J. Coates ${ }^{* a, b}$, M. Carter ${ }^{\mathrm{a}}$, C.E. Leffa, ${ }^{\mathrm{a}}$, A.D. Griffiths ${ }^{\mathrm{a}, \mathrm{b}}$, N. Schmitz ${ }^{\mathrm{c}}$, R. Jaumann ${ }^{\mathrm{d}}$, J.-L. Josset ${ }^{\mathrm{e}}$, G.

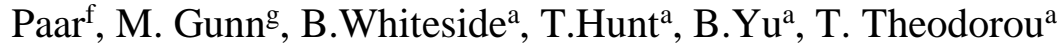 \\ and the Rosalind Franklin (ExoMars 2022) PanCam team \\ ${ }^{a}$ Mullard Space Science Laboratory, University College London, UK \\ ${ }^{\mathrm{b}}$ Centre for Planetary Science at UCL/Birkbeck, London, UK. \\ 'Institute of Planetary Research, German Aerospace Centre (DLR), Berlin, Germany. \\ ${ }^{\mathrm{d}}$ Free University of Berlin, Institute of Geological Sciences, Berlin, Germany. \\ eSpace Exploration Institute, Neuchâtel, Switzerland. \\ fJoanneum Research, Graz, Austria. \\ gDepartment of Physics, Aberystwyth University, Aberystwyth, UK.
}

\begin{abstract}
The ESA-Russia Rosalind Franklin (ExoMars 2022) rover, with its unique 2m drill, will address key questions in the search for life on Mars. PanCam will establish the surface context, with other instruments, providing geology, atmospheric science and 3D vision. PanCam uses a pair of Wide Angle Cameras (WACs), each with an 11 position filter wheel, and a High Resolution Camera (HRC). The cameras and electronics are in an optical bench that provides the interface to the rover and planetary protection. PanCam includes a calibration target mounted on the rover deck for radiometric calibration, fiducial markers for geometric calibration and a rover inspection mirror.
\end{abstract}

Keywords: ExoMars, PanCam, cameras, space, Mars.

\section{INTRODUCTION}

The search is on for evidence of life elsewhere else in the Universe. At the moment, the only life we know of is on Earth. In our solar system, the most likely places for life beyond Earth are Mars, which we discuss here, but also Jupiter's moon Europa, and Saturn's moons Enceladus and Titan, and maybe the clouds of Venus.

Mars is one of our nearest targets for finding extraterrestrial life. 3.8-4 billion years ago, Mars was much warmer and wetter, as shown by several space missions; it also had a magnetic field, volcanism and was habitable at that time.

Now, Mars is dry, has only crustal magnetic fields and extinct volcanoes. It also has a very thin carbon dioxide rich atmosphere, with only $1 \%$ of Earth's atmospheric pressure, varying daily and seasonally ${ }^{1}$. The surface is extremely harsh for life, as the temperature varies between $0-10$ degrees $\mathrm{C}$ by day and -100 to -120 degrees $\mathrm{C}$ at night. The thin atmosphere also means that the surface is bathed in harmful ultraviolet light, and the lack of a global magnetic field allows a high radiation environment at the surface, with cosmic rays from the galaxy and the Sun.

The Rosalind Franklin (ExoMars 2022) rover (see Figure 1) is the only planned mission designed to drill $2 \mathrm{~m}$ under the harsh Mars surface ${ }^{2}$, and thus has the best chance of detecting biomarkers. It will analyse samples from the sub-surface in-situ, and send data back to Earth via the ExoMars Trace 
Gas Orbiter and other orbiters, already in Mars orbit. The rover was built by Airbus Defence \& Space in Stevenage, UK, and the prime contractor for the whole mission is Thales-Alenia Space in Italy. Key mission elements, including the Kazachok landed platform, are made by Lavochkin in Russia, all overseen by ESA and Roscosmos.

\section{THE ROSALIND FRANKLIN ROVER}

The capable instrument complement includes 'context' instruments (PanCam - our scientific camera system $^{3}$ - see below), an infrared spectrometer ISEM ${ }^{4}$ for mineralogy, a ground penetrating radar WISDOM $^{5}$ for subsurface rock outcrop and water ice detection, a neutron detector ADRON 6 for sub-surface hydrogen, inferring water, and a close-up imager CLUPI ${ }^{7}$. In the tip of the drill is the miniaturised visible and infrared Ma_MISS ${ }^{8}$ instrument, for subsurface geological context. The drilled samples are analysed inside the rover with the 'analytical drawer' instruments MicrOmega', a visible-infrared spectrometer, a Raman laser spectrometer ${ }^{10}$ which does mineralogy from fluorescence, and the Mars Organics Mass Analyser MOMA ${ }^{11}$.

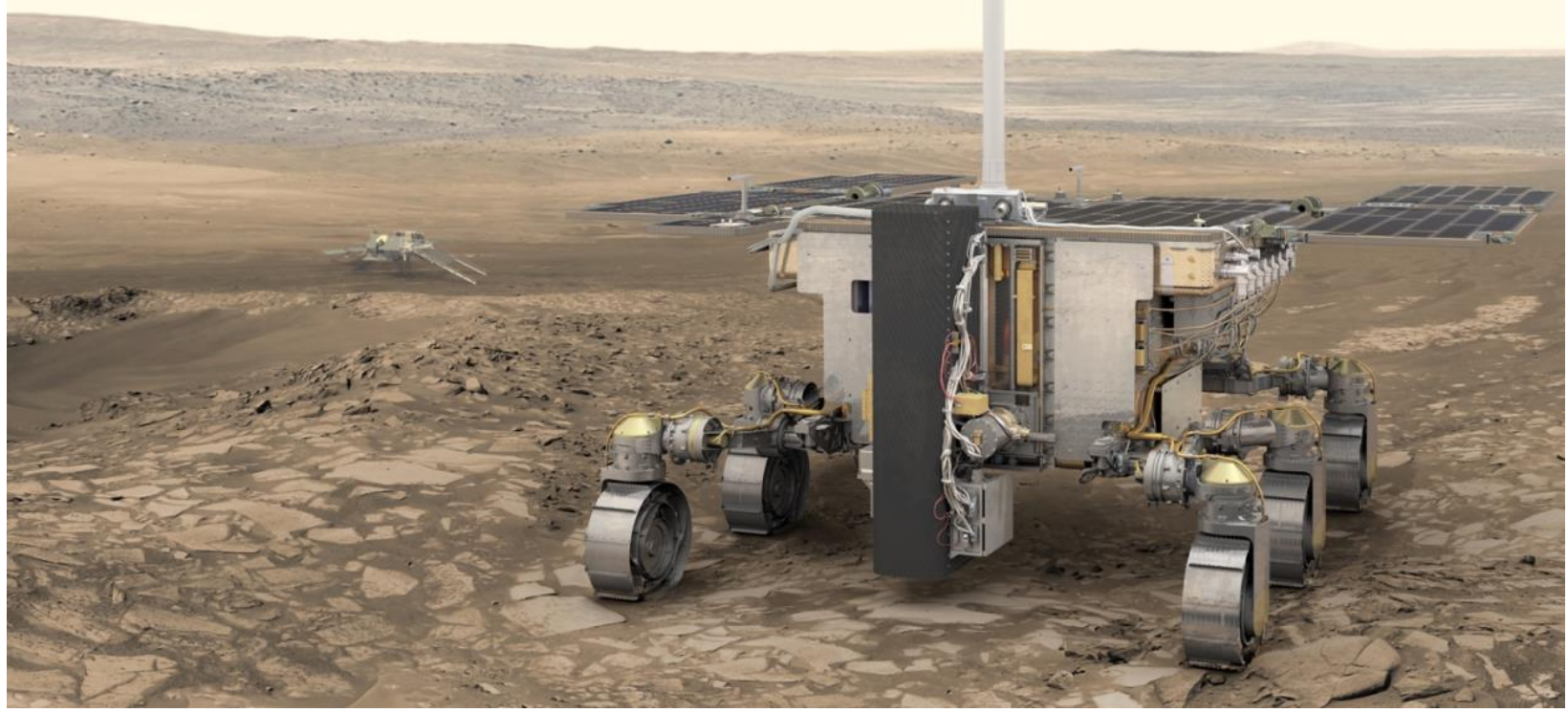

Figure 1. The Rosalind Franklin (ExoMars) rover (courtesy ESA). PanCam is at the top of the mast about $2 \mathrm{~m}$ above the Martian surface. The Kazachok landed platform is in the background.

The Kazachok landed platform also includes an excellent array of instruments for science measurements complementary with those of the rover. 
The mission is planned for launch on 20 September 2022 and landing on 10 June 2023. The lifetime is 211 'sols' (Martian days, each 24 hours and 40 minutes), so the end of mission will be approximately January 2024.

The rover is named after Rosalind Franklin, the brilliant X-ray crystallographer, whose work was critical to Watson and Crick's discovery of the double helix structure of DNA.

\section{OUR INSTRUMENT - PANCAM}

PanCam $^{3}$, the Panoramic Camera system, provides the science 'eyes' of the Rosalind Franklin rover. It consists of three cameras - two 'wide angle' cameras (WACs) and a High Resolution Camera (HRC) (see Figure 2). The instrument characteristics are summarised in Table 1.

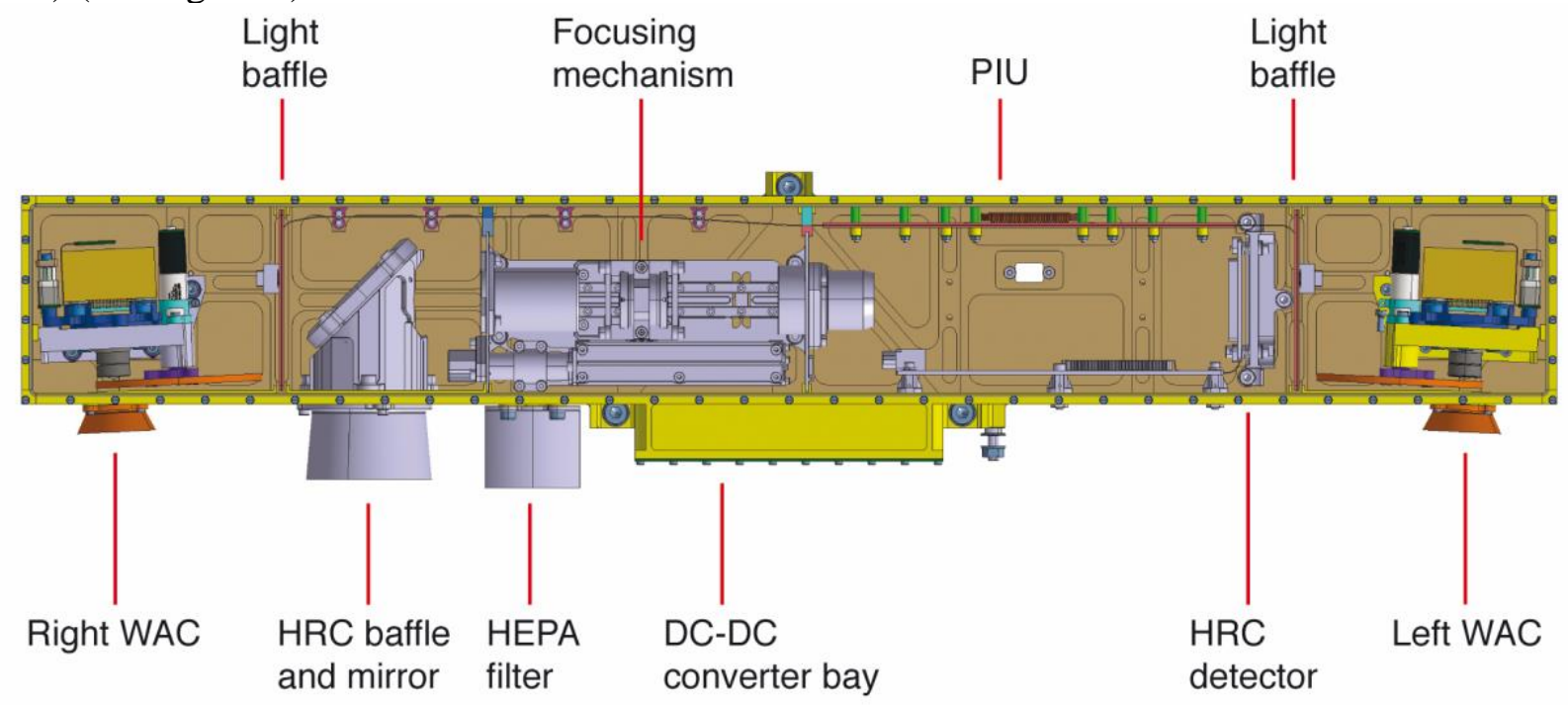

Figure 2. The PanCam optical bench $\left(\right.$ schematic $^{3}$ ). The two WACs are separated by $50 \mathrm{~cm}$. Courtesy UCL-MSSL

The separation of the two WACs is $50 \mathrm{~cm}$, providing better stereo reconstruction than possible with the human eyes, and $\mathrm{mm}$ resolution at $2 \mathrm{~m}$ (the height of the mast). Each WAC has a filter wheel with 11 filters (see Figure 3). These include broad R,G and B filters for colour, narrower geological filters for rock composition, and the narrowest are atmospheric filters which will be used to determine water abundance between the Sun and the camera. The geological filters have been selected to provide the best determination of water rich minerals using multispectral analysis ${ }^{12,13}$. The atmospheric filters will be used near Martian sunset to determine the profile of water in the atmosphere and linking with atmospheric escape.

The HRC acts like a 'telescope' to provide sub-mm resolution at $2 \mathrm{~m}$ from the camera, providing rock texture.

The optics and the electronics (a PanCam Interface Unit and a DC-DC converter) for PanCam are housed in an 'optical bench', on top of the rover's mast. This provides protection against dust and also a 'planetary protection' barrier. Cleanliness has been a key part of this mission, as we must try to avoid false life detection on Mars by taking it from Earth. 
As well as the optical bench, so-called 'small items' are also part of PanCam. These include a colour calibration target, fiducial markers and a Rover Inspection Mirror for seeing obstacles under the rover itself. With this we are able to get the combination of stereo, colours, shapes and scales.

We have a large and capable team of scientists and engineers on the team ${ }^{3}$. The hardware has come from the UK (UCL-MSSL - filter wheels, PanCam interface unit, DC-DC converter \& optical bench, and Aberystwyth - small items), with the WACs from TAS-CH in Switzerland and the HRC from DLR and OHB in Germany. 3D vision software is from JR in Austria. The science team includes experts from 9 countries, a truly international endeavour as most of the instruments on board. The mission is highly collaborative and the data from all the instruments are complementary.

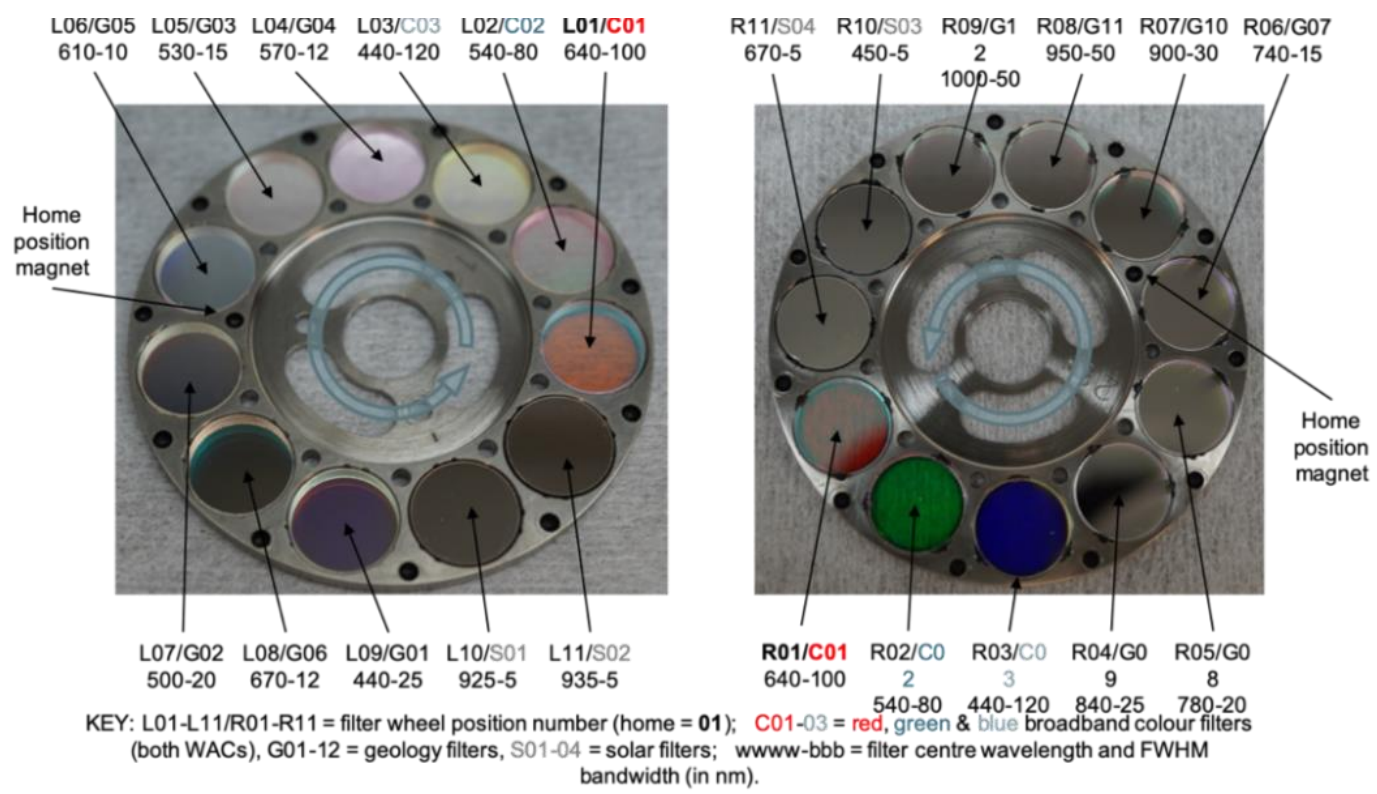

Figure 3. The flight filter wheels, showing the centre wavelength and bandwidth of each filter (nm). Courtesy UCL-MSSL

A number of field trials have tested the instrument ${ }^{14}$ and the team on Earth to make them ready for working with data from Mars, and to enable them to make quick decisions in the daily operations planning ${ }^{15}$. The rover and the mission will be guided by both science and engineering.

In August 2019, PanCam was integrated onto the rover mast (see Figure 4). It was especially gratifying at that time to see the 'first light' from PanCam on the rover, showing that everything works all the way from PanCam through the rover systems and transmitted for scientific analysis (see Figure 5). Good calibration is vital for the scientific interpretation of the data, and we made time for these measurements, both radiometric and geometric, in the tight timescale of the instrument delivery. We have also simulated the views through PanCam's scientific 'eyes' ${ }^{16}$. 
Table 1 - Main PanCam characteristics (adapted from ${ }^{3}$ )

\section{WACs (x2)}

$38.3 \times 38.3$

$1024 \times 1024$

Multispectral Filter Wheel

11 (x 2 eyes)

653

1.31

Fixed $(1.0 \mathrm{~m}-\infty)$
HRC

$4.88 \times 4.88$

$1024 \times 1024$

$\begin{array}{lll}\text { Filter type } & \text { Multispectral Filter Wheel } & \text { RGB } \\ \text { Filter number } & 11 \text { (x 2 eyes }) & \text { Bayer } \\ \text { IFOV }(\mu \mathrm{rad} / \text { pixel }) & 653 & 83 \\ \text { Pixel scale at } 2 \mathrm{~m}(\mathrm{~mm}) & 1.31 & 0.17 \\ \text { Focus } & \text { Fixed }(1.0 \mathrm{~m}-\infty) & \text { Mechanical autofocus }(0.98 \mathrm{~m}-\infty)\end{array}$

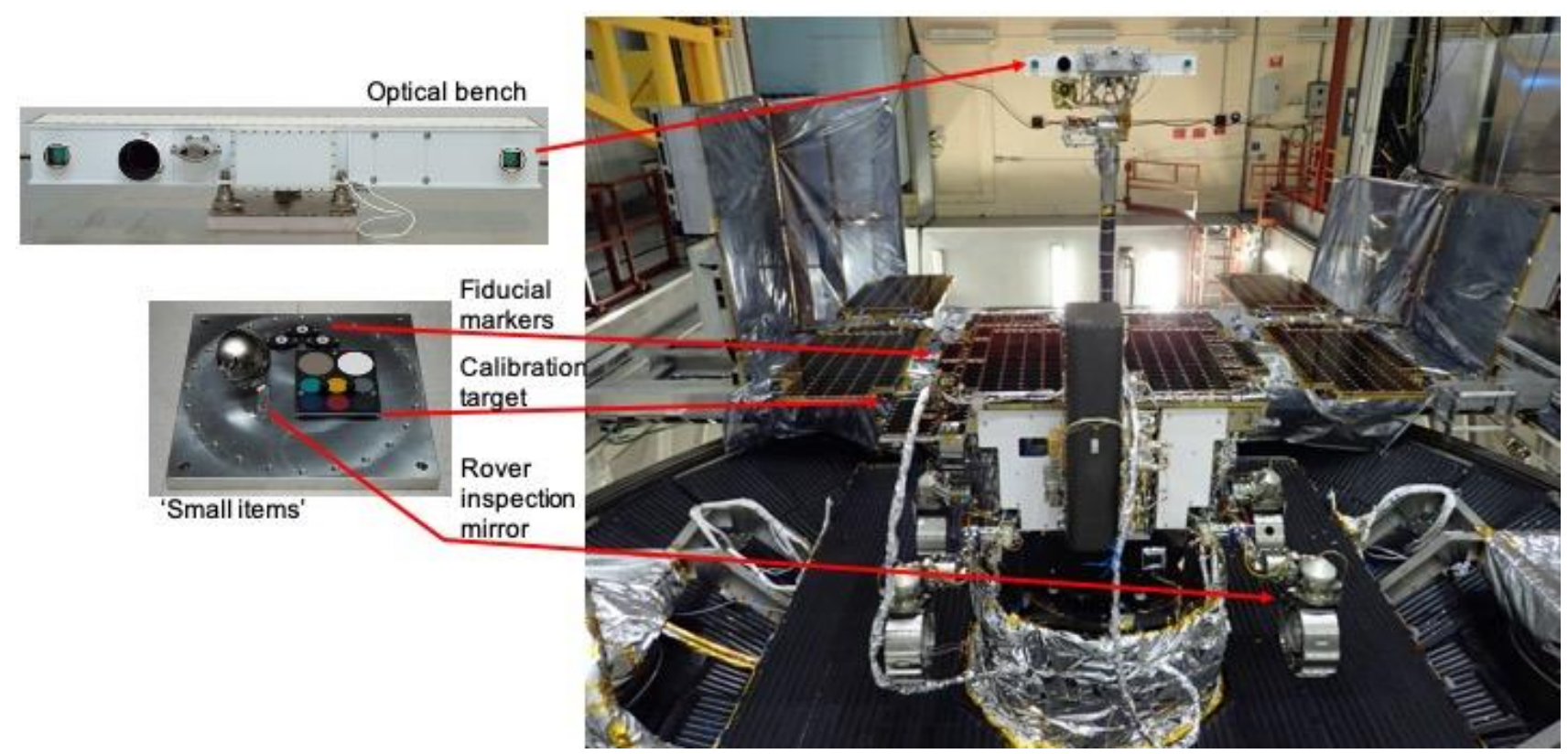

Figure 4. The flight model of PanCam on the rover, showing locations of the optical bench and 'small items'. PanCam images courtesy UCL-MSSL - M. dela Nougerede, rover images courtesy Airbus - M.Alexander 


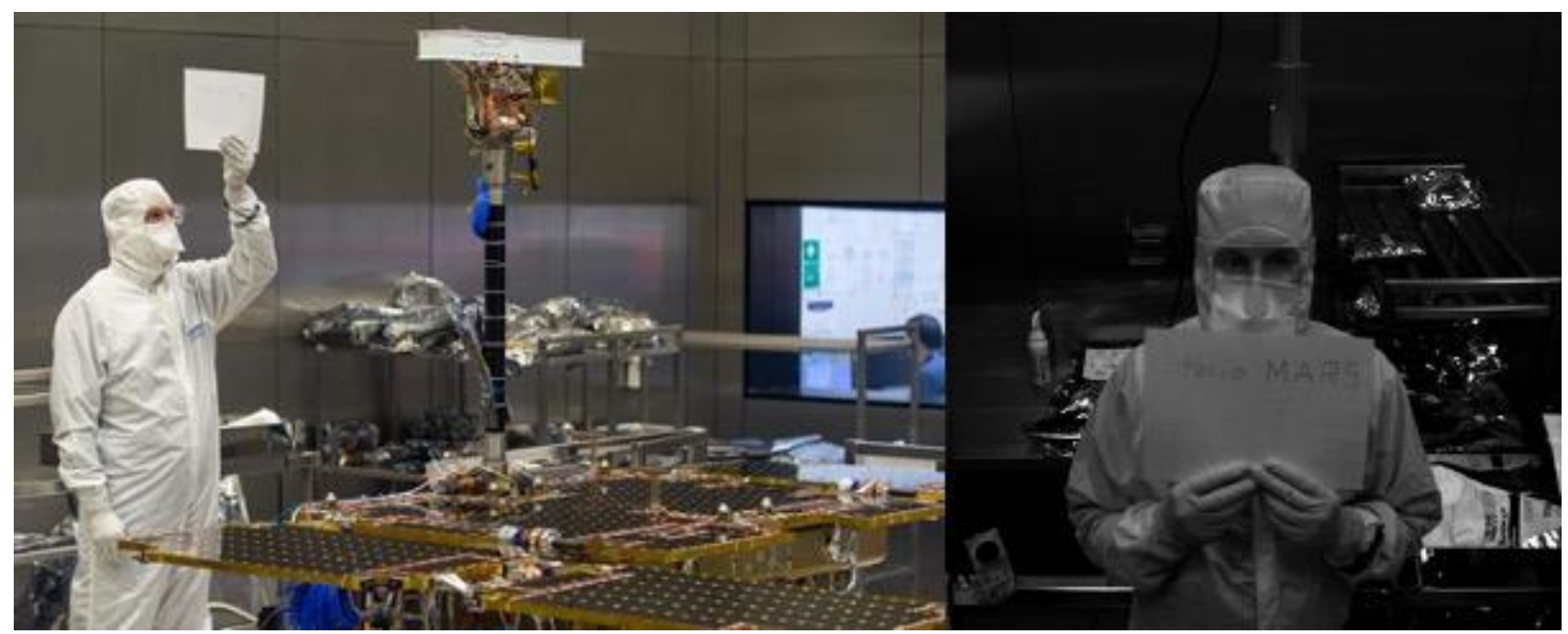

Figure 5. The 'First light' from PanCam on the rover, August 2019. (Left) courtesy Airbus -M.Alexander, (right) courtesy ESA/ExoMars/PanCam team. Matt Gunn (Aberystwyth) is shown with the rover in the clean room at Airbus.

\section{CONCLUSIONS}

To conclude, the Rosalind Franklin rover will provide an important new dimension on Mars drilling $2 \mathrm{~m}$ under the surface, the only mission planned to look for biomarkers there. This exciting mission has the best chance of finding traces of life on Mars, at least until Mars sample return missions later this decade.

PanCam, with the other context instruments, provides geological and atmospheric context for the mission. We can't wait for the launch in 2022 and landing in 2023!

\section{ACKNOWLEDGMENTS}

We thank the entire PanCam engineering team for successfully delivering PanCam, especially M. Carter, B. Whiteside, T. Hunt, C. Theobald, C. Leff, A. Griffiths (UCL-MSSL, UK); N. Schmitz, R. Jaumann, F. Trauthan (DLR-PF, D); E. Gubbini, C. Wimmer, M. Glier (OHB, D; J.-L. Josset (SEI, $\mathrm{CH}$ ); M. Mosberger (TAS-CH, CH); M. Gunn, D. Barnes ${ }^{+}$(Aberystwyth University, UK), G. Paar (Joanneum Research, A). We also thank the entire international (9-nation) PanCam science team for support. We thank the UK Space Agency, DLR Agency and ESA-Prodex for financial support.

\section{REFERENCES}

[1] Read, P. L., Lewis, S. R \& Mulholland, D. P., "The physics of Martian weather and climate: a review," Reports on Progress in Physics 78(12), 1-54 (2015).

[2] Vago, J. L., Westall, F., Coates, A. J., et al., "Habitability on Early Mars and the Search for Biosignatures with the ExoMars Rover," Astrobiology 17(6-7), 471-510 (2017). 
[3] Coates, A. J., Jaumann, R., Griffiths, A. D., et al., "The PanCam instrument for the ExoMars rover," Astrobiology 17(6-7), 511-541 (2017).

[4] Korablev, O. I., Dobrolensky, Y., Evdokimova, N., et al., "Infrared Spectrometer for ExoMars: A Mast-Mounted Instrument for the Rover,” Astrobiology 17(6-7), 542-564 (2017).

[5] Ciarletti, V., Clifford, S., Plettemeier, D., et al., "The WISDOM Radar: Unveiling the Subsurface Beneath the ExoMars Rover and Identifying the Best Locations for Drilling," Astrobiology 17(6-7), 565-584 (2017).

[6] Mitrofanov, M. L., Litvak, S. Y., Nikiforov, I., et al., "The ADRON-RM Instrument Onboard the ExoMars Rover,” Astrobiology 17(6-7), 585-594 (2017).

[7] Josset, J. L., Westall, F., Hofmann, B.A., et al., “The Close-Up Imager Onboard the ESA ExoMars Rover: Objectives, Description, Operations, and Science Validation Activities," Astrobiology 17(6-7), 595-611 (2017).

[8] De Sanctis, M. C., Altieri, F., Ammannito, E., et al., "Ma_MISS on ExoMars: Mineralogical Characterization of the Martian Subsurface," Astrobiology 17(6-7), 612-620 (2017).

[9] Bibring, J.-P., Hamm, V., Pilorget, C., et al., "The MicrOmega Investigation Onboard ExoMars, Astrobiology," 17(6-7), 621-626 (2017).

[10] Rull, F., Maurice, S., Hutchinson, I., et al., "The Raman Laser Spectrometer for the ExoMars Rover Mission to Mars", Astrobiology 17(6-7), 627-654 (2017).

[11] Goesmann, F., Brinckerhoff, W. B., Raulin, F., et al., “The Mars Organic Molecule Analyzer (MOMA) Instrument: Characterization of Organic Material in Martian Sediments," Astrobiology 17(6-7), 655-685 (2017).

[12] Cousins, C. R., Griffiths, A. D., Crawford, I. A., et al., “Astrobiological considerations for the selection of the geological filters on the ExoMars PanCam instrument," Astrobiology 10(9), 933-951 (2010).

[13] Cousins, C., Gunn, M., Prosser, B., et al., "Selecting the geology filter wavelengths for the ExoMars Panoramic Camera instrument”, Planet. Space Sci. 71(1), 80-100 (2012).

[14] Harris, J. K, Cousins, C. R., Gunn, M., et al., "Remote detection of past habitability at Marsanalogue hydrothermal alteration terrains using an ExoMars Panoramic Camera Emulator," Icarus 252, 284-300 (2015).

[15] Balme, M. R., Curtis-Rouse, M. C., Banham, S., et al., "The 2016 UK Space Agency Mars Utah Rover Field Investigation (MURFI)", Planet. Space Sci., 165, 31-56 (2019). 
[16] Miles, H. C., Gunn M. D. \&. Coates, A. J., "Seeing through the 'Science Eyes' of the ExoMars Rover,” IEEE Computer Graphics \& Applications 40(2), 71-81 (2020). 